\title{
Twisted photons: new quantum perspectives in high dimensions
}

\author{
Manuel Erhard ${ }^{1,2}$, Robert Fickler ${ }^{3}$, Mario Krenn $^{1,2}$ and Anton Zeilinger ${ }^{1,2}$ \\ Twisted photons can be used as alphabets to encode information beyond one bit per single photon. This ability offers great \\ potential for quantum information tasks, as well as for the investigation of fundamental questions. In this review article, we give \\ a brief overview of the theoretical differences between qubits and higher dimensional systems, qudits, in different quantum \\ information scenarios. We then describe recent experimental developments in this field over the past three years. Finally, we \\ summarize some important experimental and theoretical questions that might be beneficial to understand better in the near \\ future.
}

Light: Science \& Applications (2018) 7, 17146; doi:10.1038/lsa.2017.146; published online 9 March 2018

Keywords: entanglement; high-dimensional quantum systems; orbital-angular-momentum of photons; quantum communication

\section{INTRODUCTION}

Quantum information science and quantum information technology have seen a virtual explosion worldwide. The explosion is all based on the observation that fundamental quantum phenomena on the individual particle or system-levels lead to completely novel ways of encoding, processing and transmitting information. Quantum mechanics, a child of the first third of the 20th century, has found numerous realizations and technical applications, many more than were thought possible at the beginning. Decades later, it became possible to do experiments with individual quantum particles and quantum systems. This was due to technological progress, and particularly for light, the development of the laser. Only about 40 years ago, quantum phenomena were studied for their own curiosities. The motivation was that the predictions of quantum mechanics for individual systems were rather counterintuitive. People wanted to see these in a concrete way in the laboratory. Fundamental phenomena include quantum superposition for individual particles, as corroborated for example by neutron interferometers and by the double slit experiment for individual massive particles and for individual photons, the objective randomness of the individual quantum event, quantum entanglement ${ }^{1}$ and entanglement as signified by the Einstein-Podolsky-Rosen paradox ${ }^{2}$ and Bell's theorem ${ }^{3}$ and the nocloning theorem ${ }^{4}$. To date, these fundamental experiments in the new field of quantum information science and quantum information technology were signified by such notions as quantum communication, quantum cryptography, quantum teleportation, quantum computation and many more. These encompass a broad understanding that all this will lead to a future of new information technology.
Hitherto, nearly all experiments and also nearly all realizations in the fields just mentioned have been performed with qubits, which are two-level quantum systems. We suggest that this limitation is again mainly a technological one, because it is very difficult to create, manipulate and measure more complex quantum systems.

Here we provide a specific overview of some recent developments with higher-dimensional quantum systems. We mainly focus on Orbital Angular Momentum (OAM) states of photons and possible applications in quantum information protocols. Such states form discrete higher-dimensional quantum systems, also called qudits. A qudit can be seen as a quantum particle which is not limited to two states but in principle can have any number of discrete levels.

Specifically, we will first address the question what kind of new fundamental properties exist and the quantum information applications, which are opened up by such novel systems. Then, we present an overview of the recent developments in the field by discussing several notable experiments over the past 2-3 years. Finally, we conclude with several important open questions which will be interesting for investigations in the future.

Due to limitation of space, we cannot cover the breakthrough experiments in the area of high-dimensional quantum information memories ${ }^{5,6}$, in high-dimensional quantum information processing with other physical systems ${ }^{7-10}$, coherent transitions of atomic states driven by the OAM of photons ${ }^{11}$ or the novel methods to manipulate OAM of light that may improve future quantum experiments such as the plasmonic q-plates ${ }^{12}$ and light carrying OAM produced in waveguide chips ${ }^{13}$.

\footnotetext{
${ }^{1}$ Vienna Center for Quantum Science \& Technology (VCQ), Faculty of Physics, University of Vienna, Boltzmanngasse 5, Vienna 1090, Austria; ${ }^{2}$ Institute for Quantum Optics and Quantum Information (IQOQI), Austrian Academy of Sciences, Boltzmanngasse 3, Vienna 1090, Austria and ${ }^{3}$ Department of Physics, University of Ottawa, Ottawa, Ontario K1N 6N5, Canada

Correspondence: M Erhard, Email: manuel.erhard@univie.ac.at

Received 27 July 2017; revised 21 September 2017; accepted 16 October 2017; accepted article preview online 17 October 2017

The accepted article preview was available with the details: Light: Science \& Applications (2018) 7, e17146; doi: 10.1038/lsa.2017.146
} 
As we focus here on the very recent developments in this field, we have to leave out many important historic experiments. For a compact overview of research in structured light, we refer to Refs 14,15. Readers interested in the general developments in quantum science with OAM of photons, we would like to refer to a review from 2007 Ref. 16 and from 2017 Ref. 17. Readers interested in the theoretical foundations of quantum information may look into Refs 18-21, and to get an overview of photonic quantum information one may look into Ref. 22.

\section{OAM OF PHOTONS}

Photons are excellent for quantum communication as they can be transmitted over long distances with very low loss and without any known decoherence mechanism in free space ${ }^{23}$. A special property of photons is their orbital angular momentum ${ }^{24}$. Quantum mechanically, the orbital angular momentum occurs in discrete steps of $\ell \hbar$, where $\ell$ is an in principle unbounded integer. Therefore, high-dimensional quantum information can be stored in the orbital angular momentum of single photons. The fundamental property of photons carrying OAM is the presence of the helical phase factor $e^{i \ell \phi}$ where $\phi$ is the azimuthal coordinate that is in the plane transverse to the propagation direction.

A variety of spatial modes that carry OAM exist, sharing a helical phase factor but differing in their radial structure. The most prominent example, the Laguerre-Gauss mode $L G_{p}^{\ell}$, accurately describes a photon carrying an OAM of $\ell \hbar$. Here, $p$ is the radial quantum number. Figure 1 shows experimental images of single photons with different OAM values. The defining feature of LG modes is their spatial phase pattern. Specifically, the pattern is a helical phase that wraps around the axis of propagation $\ell$ times within a wavelength, which results in a phase singularity at the beam center. Thus, the intensity profile of an LG mode shows the typical donut shaped structure. For single photons, the intensity profile gives the probability of detecting a photon at a certain point. If we place a triggered single-photon camera and detect many heralded photons ${ }^{25}$, then the intensity profile of the respective LG mode emerges, which is shown in the filmstrips in Figure 1. A few examples of higher order OAM modes, which can be used to encode high-dimensional qudit states, together with some exemplary superposition structures are shown in Figure 2.

\section{ADVANTAGES OF HIGHER-DIMENSIONAL QUANTUM SYSTEMS}

\section{Higher information capacity}

The first and rather obvious benefit of qudits is the enlarged information content that a single quantum carrier can transmit. Any additional orthogonal state used in the encoding scheme increases the information encoded in a single quantum system. A simple example would be the use of ququarts, that is, four dimensional systems, which can be used to encode 2 bits of information: $|0\rangle=00,|1\rangle=$ $01,|2\rangle=10$ and $|3\rangle=11$. The often used measure of information capacity, which is given in $\log _{2}(d)$ bits/photon, can be seen as a measure of how many classical bits have to be used to be able to encode the same amount of information. In principle there is no upper limit of $d$ (Except of a potential limit which arises due to the finite size of the universe), which means that a single quantum system, like a photon, has the potential to encode an arbitrarily large amount of information.

Enhanced robustness against eavesdropping and quantum cloning Although the perfect copying of a quantum state is impossible due to the no-cloning theorem, it was discovered that it is possible to approximatively clone a quantum state ${ }^{4}$. This so-called optimal cloning can be achieved with a cloning machine wherein one photon is cloned to two imperfect copies. Here, the cloning fidelity $F_{\text {clon }}^{d}$, (the overlap of the clones and the original state), is limited to the upper bound of $F_{\text {clon }}^{d}=\frac{1}{2}+\frac{1}{1+d}$. This dependence on the dimensionality shows that the fidelity of the cloned qubits can be as high as $F_{\text {clon }}^{2}=\frac{5}{6}$, while states with higher dimensionalities $d$ can only be cloned with lower fidelities down to $50 \%$ in the limit of $d \rightarrow \infty$. This reduction of the cloning fidelity for qudits nicely depicts the superiority of highdimensional quantum cryptography and the increased error thresholds as it is more difficult for an eavesdropper to hack a high-dimensional key distribution scheme.

Hence, in addition to the increased information capacity, quditstates are more robust to background noise and hacking attacks when used in quantum cryptography 26,27 . To ensure the security of an established quantum link, which can then be used to share a random encryption key, it is essential to stay below a certain error threshold. When this error bound is met, no eavesdropper could have attacked
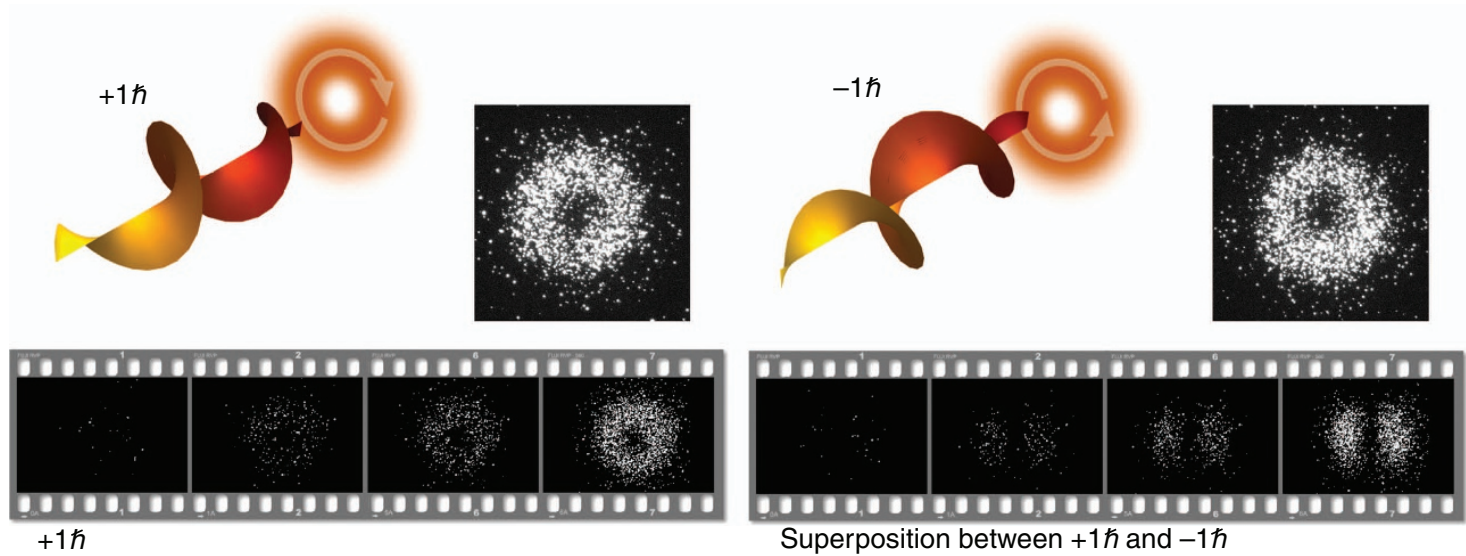

Figure 1 Orbital angular momentum (OAM) of twisted photons. A helical (twisted) phase $e^{i \ell \phi}$ structure leads to a quantized amount of $\ell \hbar$ of OAM. Upper row: Photons with $+1 \hbar$ of OAM have a phase structure that varies azimuthally from 0 to $2 \pi$, which also leads to a vortex along the beam axis and thus, an intensity null. The black/white images are built by accumulating many single photon events. Lower row: Accumulation of approximately $30,250,600$ and 2500 photons (from left to right) to reveal the intensity structure of an $|+1\rangle$ OAM mode and the superposition between $+1 \hbar$ and $-1 \hbar$. The images have been recorded with a single-photon sensitivity low-noise ICCD camera ${ }^{25}$. 


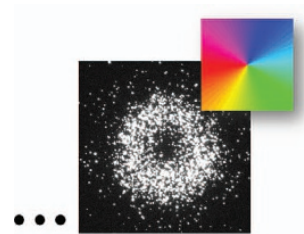

$|-1 \hbar\rangle$

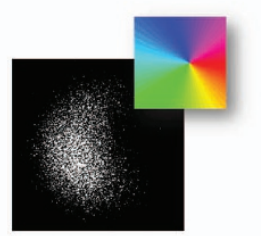

$|0 \hbar\rangle+|+1 \hbar\rangle$

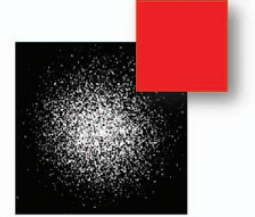

$|0 \hbar\rangle$

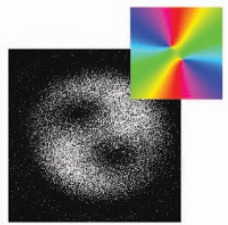

$|0 \hbar\rangle-i|+2 \hbar\rangle$

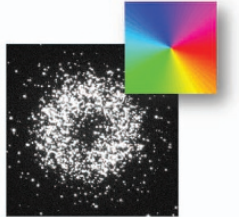

$|+1 \hbar\rangle$

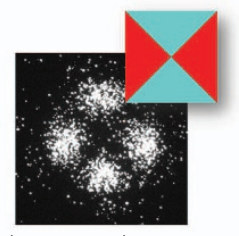

$|+2 \hbar\rangle+|-2 \hbar\rangle$

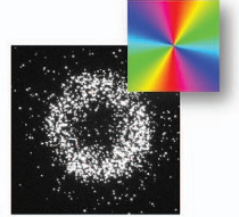

$|+2 \hbar\rangle$

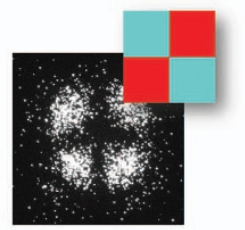

$|+2 \hbar\rangle-|-2 \hbar\rangle$

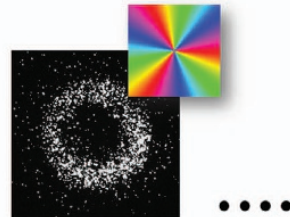

$|+3 \hbar\rangle$

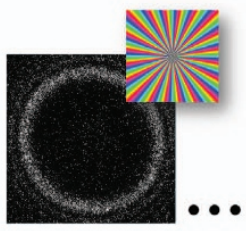

$|+20 \hbar\rangle$

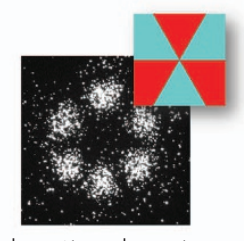

$|+3 \hbar\rangle+|-3 \hbar\rangle$

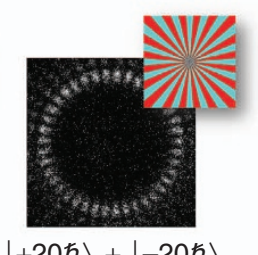

$|+20 \hbar\rangle+|-20 \hbar\rangle$

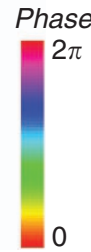

0

Figure 2 Single-photon images of higher order OAM states (upper row) and superposition states (lower row). Each mode can be used to increase the dimensionality of the qudit state, for example, the subset modes in the upper row from $-1 \hbar$ to $+3 \hbar$ could describe a five-dimensional state. Quantum experiments also require coherent superposition states; a few examples of specific modes are shown in the lower row. All insets display the underlying phase structures in the color scales shown on the lower right.

the transmission and security is ensured by quantum physical laws. It is important to note, that it does not matter if an actual eavesdropper had access or the errors were caused by strong noises. This feature of quantum cryptography holds for both entanglement based systems ${ }^{28}$ as well as prepare-and-measure QKD schemes ${ }^{29,30}$. For qubit systems, this bound has been proven to be as low as approximately 11 or $12.6 \%$ if two or three mutually unbiased bases are taken into account, respectively ${ }^{31-33}$. However, if higher-dimensional quantum states are used, this requirement can be weakened, for example, with ququarts already up to nearly $19 \%$ or $22 \%$ errors can be tolerated (again, for two or three mutually unbiased bases), respectively ${ }^{27,32,34,35}$. This threshold can be as large as $50 \%$ for an infinite dimensional state, a behavior for which optimal cloning already gave a hint.

Quantum communication without monitoring signal disturbance It was recently discovered that high-dimensional systems can also be beneficial for prepare-and-measure quantum cryptography applications if only qubits are encoded and are somewhat hidden in an enlarged high-dimensional Hilbert space. The two-dimensional quantum information, which after successful performance of the protocol is used to establish a secure key, is encoded in the relative phases between the utilized high-dimensional states.

It was proven that after a clever randomized measurement at the receiver and a large enough Hilbert space, the monitoring of the signal disturbance can be neglected ${ }^{36}$, in contrast to other prepare-andmeasure protocols such as the seminal BB84 (Ref. 29). In simplified terms, this means that renouncing the advantage of a large information capacity per photon and still using complex qudit states facilitates a secure transmission in highly noisy channels.

Larger violation of local-realistic theories and its advantages in quantum communication

John Bell showed in 1964 that the predictions of specific quantum mechanical measurements on qubits cannot be explained in a local realistic manner ${ }^{3}$. Local realism is the worldview in which physical properties of objects exist prior to and independent of the measurement, and physical influences are bounded by the speed of light. A violation of Bell's inequality (which have been demonstrated recently in a loophole-free way ${ }^{37-40}$ ) shows that such a worldview can no longer be maintained in the face of experimental evidences.
First generalizations to multilevel systems in the early 1980s by Mermin and Garg ${ }^{41,42}$ showed that the conflict between local realism and quantum mechanics diminishes for larger dimensions. Interestingly, those results were interpreted as a necessary emergence of local realism in the classical limit, or a quantum-to-classical transition for large quantum numbers. A decade later, in 1992, Peres was able to show for the first time a violation of local realism that is constant for arbitrary large dimensions ${ }^{43}$. It took nearly another decade until Kaszlikowski et $a l^{44}$ showed the first high-dimensional violation of local realism that is larger than those in two dimensions (a larger violation of Bell type inequalities means that the violation is more robust against noise). In Ref. 44 it is shown that the maximum tolerable noise, which still allows a violation of local-realism, increases with the dimension because the Bell inequality is stronger violated. Their numerical approach showed that for $3 \leq d \leq 9$, Bell-type inequalities can be found where the violation of local realism increases as the dimension of the system increases. Soon afterwards, inequalities for violating local realism for arbitrarily high-dimensional systems were found by Collins and colleagues (often referred to as CGLMP inequality nowadays $)^{45}$. While analytical results are still missing, numerical estimates suggest that the violations increase monotonically with the number of dimensions ${ }^{44,46}$.

The violation of local realism is not only interesting for fundamental reasons but has direct applications in quantum communication protocols. These advantages go beyond the more stringent cloning fidelities discussed in the Section of "Enhanced robustness against eavesdropping and quantum cloning". In entanglement-based QKD protocols, the presence of an eavesdropper is excluded by violating a local realistic inequality. As higher-dimensional systems allow for larger violations, those systems can supersede two-dimensional implementations of QKD. Analogous as before, one can imagine situations where qubits cannot be used to generate a secure key, whereas a high-dimensional system still enables to securely distribute an encryption key. We explain this now in two concrete examples.

Huber and Pawlowsky ${ }^{47}$ investigated an entanglement-based quantum key distribution scenario ${ }^{28}$. In this scenario, Alice and Bob measure entangled photon pairs, which they use to create the key and verifying the security of the transmission. To generate the key, they both measure in perfect correlations. To verify the security, Bell measurements need to be performed, such as $\mathrm{H}+22.5^{\circ} / \mathrm{V}, \mathrm{H}-22.5^{\circ} / \mathrm{V}$, 
$\mathrm{D}+22.5^{\circ} / \mathrm{A}, \mathrm{D}-22.5^{\circ} / \mathrm{A}$, with horizontal $(\mathrm{H})$ and vertical $(\mathrm{V})$, diagonal $(D=1 / \sqrt{2}(H+V))$ and anti-diagonal $(A=1 / \sqrt{ } 2(H-V))$ polarisation. If the eavesdropper knows when the Bell measurement is performed, she would not look at those photons (to not disturb them), and only would look at the photons for the generation of the secret key. Therefore, the choice when the Bell measurement is performed has to be random. If the randomness is weak, the eavesdropper could still try to hide her appearance ${ }^{48}$. Above a certain threshold, weak randomness therefore prevents a secure quantum key distribution. Interestingly, because the violation of high-dimensional Bell inequalities can be much stronger than two-dimensional violations, the threshold of acceptable loss of randomness is significantly larger for high-dimensional systems. This leads to the situation where two-dimensional quantum key distribution (for instance, performed with polarization of photons) is hopeless while high-dimensional systems would still permit a secure channel ${ }^{47}$.

Device-independent quantum key distribution (DI-QKD) requires the detection of a large fraction of the distributed entangled states. The smallest known fraction for closing the so-called detection loophole is $66.7 \%$, based on the non-maximally entangled states as shown by Eberhard $^{49}$. Reaching this bound is quite challenging for photons, and has only been exceeded in 2013 Refs 50,51. However, it was shown by Vertesi, Pirono and Brunner that for $d=4$, the detection efficiency required for overcoming the detection loophole can drop by almost $5 \%$ to $61.8 \%$ (Refs 52,53). Surprisingly, the results for larger dimensions are not known so far.

\section{Quantum computation with qudits}

Using qudits can also have advantages in quantum computing. For example, it has been shown that the distillation of important resource states for quantum computations (so-called Magic states) can be done in a way that have no direct analogue for qubits and can be several orders of magnitude more efficient ${ }^{54}$. In another approach Bocharov and colleagues investigate the Shor algorithm with qutrits, and find the promising result that qubits encoded in the slightly larger Hilbert space of a qutrit allow for a more efficient circuit structure than using natural qutrit gates ${ }^{55}$. Investigations into high-dimensional quantum computation is still an active field of research.

\section{RECENT DEVELOPMENTS IN HIGH-DIMENSIONAL QUANTUM INFORMATION WITH OAM}

After having discussed a few advantages that qudit systems offer, we will now turn to recent developments of the broad and vivid activity of high-dimensional quantum information using the OAM of photons.

\section{Creation of high-dimensional entanglement}

Photon pairs entangled in their orbital angular momentum can be produced using a spontaneous parametric down-conversion process in a nonlinear crystal $^{56}$. Due to the conservation of OAM (when the pump photon has $\ell_{p}=0$ ), the two photons have opposite OAM values $\ell_{s}=-\ell_{i}$ such that the resulting two-photon state can be written as

$$
\begin{aligned}
|\psi\rangle & =a_{0}|0\rangle_{s}|0\rangle_{i}+a_{1}|1\rangle_{s}|-1\rangle_{i}+a_{-1}|-1\rangle_{s}|1\rangle_{i}+\cdots \\
& =\sum_{\ell} a_{\ell}|\ell\rangle_{s}|-\ell\rangle_{i}
\end{aligned}
$$

where $a_{\ell}$ are coefficients that depend on the focusing parameters, phase matching conditions and crystal dimensions. This process was employed in the first quantum entanglement experiment with $\mathrm{OAM}^{56}$ and has been the workhorse for nearly all quantum experiments using
OAM entanglement as one gets high-dimensional entanglement nearly for free.

A stringent limitation, however, is that there are only very few methods to tune the parameters $a_{\ell}$. These parameters decrease significantly for higher-order modes, as has been investigated in detail by Miatto et $a^{57}$. Experimentally, it has been shown that by choosing the focusing parameters in an optimal way, one can increase the higher-order modes ${ }^{58}$, although not to a flat distribution. As an effect, the resulting entangled state is not maximally entangled. Although this state might be required for certain protocols (such as a highdimensional version of Hardy's ladder proof, which was recently investigated theoretically and experimentally to demonstrate entanglement ${ }^{59,60}$ ) it often limits the usefulness of the entangled state in quantum experiments.

An interesting recent advancement has been introduced by Zhang et $a^{61}$. They show how the usage of two-photon interference (HongOu-Mandel effect ${ }^{62}$ ) can be used to generate a large set of highdimensional states using quantum state filtering.

In another experiment, high-dimensional entanglement was generated, first in the path degree of freedom, which is simpler to custom tailor than OAM entanglement ${ }^{9}$, and afterwards transferred to OAM states $^{63}$. Because the path encoded states are compatible with integrated optics, this technique might also serve as an interface between waveguide structures and OAM modes of photons.

Another alternative approach that allows for the generation of arbitrarily, high-dimensional two-photon states has been recently proposed $^{64}$. The idea is that the creation of a pair of photons occurs in a coherent superposition of several crystals. Between the crystals, the mode number, phase and amplitude can be adjusted arbitrarily. The generation of an arbitrary four-dimensional state is shown in Figure 3 as an example. In general, $d$ crystals are pumped coherently such that one pair of photons is created in each crystal. As it is not known in which of the $d$ crystals it is created, the state is in a coherent superposition of being created in either of them. The manipulation of the mode number and phase between the crystals allows for the creation of arbitrary two-photon entangled states for example, a $d$-dimensional Bell state. As only the Gaussian mode of the downconverted photons are used, the count rates of this method can be significantly larger than those for other schemes. The same method can also be used to create very general high-dimensional multipartite entangled states ${ }^{64}$ with interesting connections to Graph Theory ${ }^{65}$. Interestingly, the method was discovered using a computer algorithm which automatically designs new quantum experiments ${ }^{66}$.

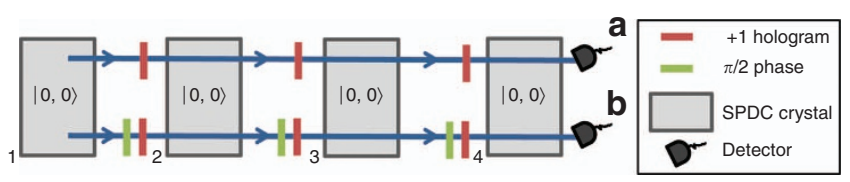

Figure 3 An experimental scheme for creating a four-dimensionally entangled two-photon state, based on entanglement by path identity ${ }^{64}$. Four crystals are pumped coherently and the paths of the down- converted photons are set to be identical. If the experimental conditions are such that there is, in principle, no information about the photon pairs origin (that is, in which of the four crystals it was created), then the pair is in a coherent superposition of being created in either of them. One can then manipulate the mode number and phase of the individual terms in the state. In this example, a hologram between each crystal shifts the mode by one and a phase of $\pi / 2$ is added such that the resulting state is a four-dimensional Bell state $|\psi\rangle=\frac{1}{2}(|0,0\rangle+i|1,1\rangle-|2,2\rangle-i|3,3\rangle$,$) .$ 


\section{Unitary transformations}

While the generation of entangled photons with OAM is welldeveloped and in the focus of different research efforts, the capability of transforming modes, which is essential for quantum informational tasks, is still in its infancy.

In contrast to the path-encoding of photonic qudits, where reprogrammable chips exist that can perform arbitrary unitary operations $^{9,67}$, for OAM only a limited set of transformations are known.

Next we present three recent, experimentally feasible approaches to perform arbitrary unitary transformations with the OAM states of single photons.

The first proposed idea we discuss here is from 2010 and uses a succession of reflections from a programmable deformable mirror device ${ }^{68}$. By using a feedback signal and a stochastic optimization, it was shown that already after three reflections a good transformation between a Gaussian mode and a third order Hermite-Gauss mode can be achieved. It will be interesting to see whether this approach can be used to perform mode-dependent transformations with precise control over phases at the quantum level.

A second, more recent technique might be another promising candidate to realize general transformations on OAM states. It has been demonstrated that using controlled random scattering, that is, properly shaping the wavefront before a strong scattering process, sorting of arbitrary spatial mode structures (including radial $p$ modes) can be achieved in a coherent way ${ }^{69}$. As such a task can be considered a complex transformation between OAM and paths, coherent transformations between different spatial modes are expected to be similarly feasible and are currently investigated. Improvements on the overall losses of the process as well as the possible limitation of mode transformations will be an important next step in order to successfully apply this technique in quantum experiments.

Third, a possible route to a multiport device for OAM modes is to build the required unitary transformation out of basic components. Here, the unitary operation that permutes all utilized OAM states in a cyclic manner, that is, a cyclic ladder transformation or highdimensional X-gate, serves as part of the building blocks for a general device. Together with the high-dimensional Z-gate, which introduces mode dependent phases, any unitary transformation can be constructed $^{70,71}$. The generalized, high-dimensional Pauli (well-known from the 2-dimensional space) operators are defined in the following way:

$$
\begin{gathered}
\hat{Z}=\sum_{n=0}^{d-1}|n\rangle \omega^{n}\langle n| \\
\hat{X}=\sum_{n=0}^{d-1}|n \oplus 1\rangle\langle n| \\
\hat{Y}=\hat{X} \hat{Z}
\end{gathered}
$$

where $\omega=e^{\frac{2 \pi i}{d}}$ and $\oplus$ is the modulo-addition. While the Z-gate for OAM modes directly corresponds to a rotation and thus can be realized by a single optical device ${ }^{72,73}$, the general experimental implementation for a cyclic transformation is not known yet. However, for the dimensions $d=3$ to 6 and $d=8$, an all-optical linear setup has been found ${ }^{66,74}$ and was recently implemented ${ }^{71,75}$. A combination of several of these basic gates are required for arbitrary transformations, thus integrating them in a stable manner will be required. Furthermore, X-gates in other dimensions than those found will be required to exploit the full capability of the highdimensional space.

\section{Optimal quantum cloning}

The experimental procedure for cloning of qubits was established in different experiments more than a decade ago $^{76}$, no high-dimensional state has been optimally cloned until recently ${ }^{77,78}$. The experiments use two-photon Hong-Ou-Mandel interference ${ }^{62}$ of OAM modes at a beam splitter ${ }^{79}$ to achieve optimal cloning of high-dimensional OAM states up to the dimension 7 (Ref. 78). It was not only shown that it is possible to clone any qudit state from any mutually unbiased bases, hence the cloning machine is universal, but it was also demonstrated that the measured cloning fidelities follow the formula described in the Section of "Enhanced robustness against eavesdropping and quantum cloning" and hacking a high-dimensional QKD scheme with a cloning attack does result in larger errors, thus making it easier to detect.

\section{Long distance high-dimensional QKD}

A promising application of high-dimensional OAM states is their use in quantum cryptography schemes. While many proof-of-principle experiments have been conducted successfully in laboratories around the world ${ }^{80-83}$, current research focuses on bringing the technology closer to real world applications by enlarging the distances over which secure keys can be distributed. Specially developed vortex fibers ${ }^{84}$ might enable fiber networks that rely on OAM encoding, however, the implementation in quantum experiments has not been demonstrated yet. Moreover, expanding such networks to global distances might be facilitated via free space links. Here, qudits help to increase data rates as well as lower the requirements for correction of induced errors, both being strongly required in outdoor environments due to inherent losses and background noise. After recent successful tests of classical communication schemes using spatially structured light over distances of up to $143 \mathrm{~km}$ (Refs 85-89), first free-space quantum experiments using spatial structures have been performed. In the first of these experiments, photons entangled in their OAM degree of freedom have been sent over an intra-city free-space link over $3 \mathrm{~km}$ in Vienna ${ }^{90}$. While it was possible to demonstrate the survival of the OAM entanglement for photons up to $\ell=|2|$, the quantum states were also only two-dimensional.

Recently, the first high-dimensional quantum states have been used to perform a free-space intra-city QKD experiment over $300 \mathrm{~m}$ in Ottawa (see Figure 4$)^{91}$. Four-dimensional quantum states, that is, ququarts, were imprinted on heralded single photons using a combination of OAM and polarization. The photons were then transmitted from Alice to Bob following the standard highdimensional BB84-protocol ${ }^{29}$. Although moderate turbulence was observed, a coupling to single mode fibers, which is required to measure the received state, was achieved with up to $20 \%$ efficiency. More importantly, an uncorrected error rate of approximately $14 \%$ was found, corresponding to 0.39 bits per sifted photon, which is well below the threshold of $18 \%$ for ququarts. The additional transmission of the heralding photon from Alice to Bob and its usage to gauge time periods of weak turbulences has further improved the extracted key rate to 0.65 bits per sifted photon. On one hand, the results show that free-space QKD with spatially structured photons is feasible and highdimensional states can be superior to qubit encoding. On the other hand, they also indicate that the extension to larger distances as well as higher data rates will require the implementation of adaptive optics to compensate for the detrimental effects of atmospheric turbulences ${ }^{92}$. Hence, the next steps will be to develop appropriate compensation schemes, to test quantum states of higher dimensions, for example 


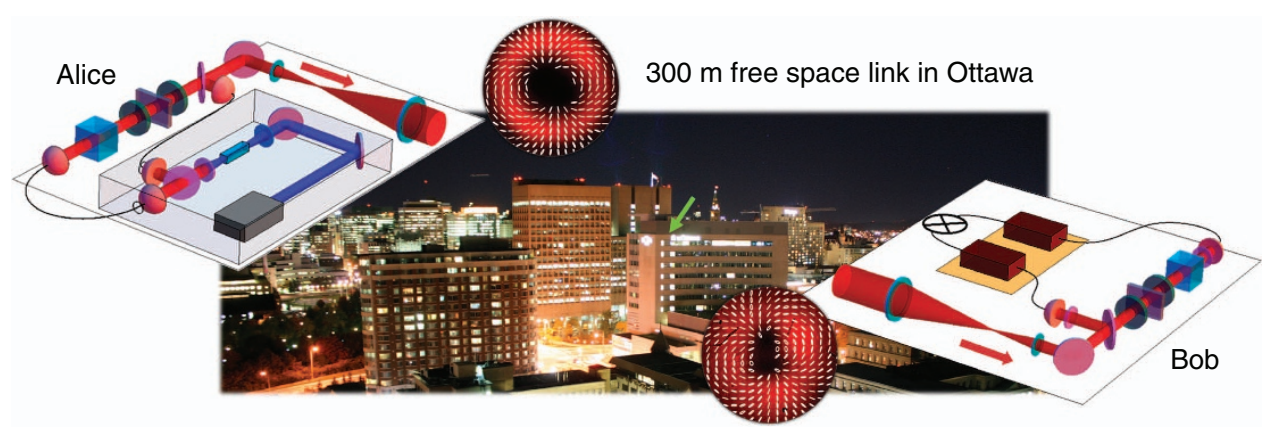

Figure 4 High-dimensional QKD over a $300 \mathrm{~m}$ long intra-city link in Ottawa ${ }^{91}$. Alice prepares non-degenerate pairs of photons in a down conversion process and splits them depending on their wavelength (gray box). Ququart states imprinted on the signal photon are prepared by means of an appropriate set of a polarizing beam splitter (cube), wave plates (green) and a q-plate (violet). A recording of an example state with a complex polarization pattern is shown in the upper inset. The two photons are then recombined and sent to Bob via the moderate turbulent link over $300 \mathrm{~m}$. Bob splits the two photons by their wavelength, measures the received state of the signal photon with same set of devices (received pattern shown in the lower inset) and heralds its arrival by detecting the idler photon. An uncorrected error rate of $14 \%$ was found corresponding to 0.39 bits per photon, which can only be considered to be secure for high-dimensional states, as the threshold for qubits is $11 \%$ (for ququarts, the bound is $18 \%$ ). The background photo shows a night view of Alice's building seen from Bob's receiving station, the green arrow points to the actual location of the sender.

with pure OAM encoding, and distribute high-dimensionally entangled photons for large quantum networks.

While all of these experiments have been performed at night, in 2002, it was shown how quantum key distribution can be performed during the day ${ }^{93}$. Very recently, a daytime quantum experiment has been performed over very loss-prone channels, indicating that even daytime satellite QKD might be possible ${ }^{94}$. Similar experiments with high-dimensional systems would be very interesting.

\section{Quantum walk}

Another exciting example that shows the advantages of OAM implementations over other degrees-of-freedom, is the implementation of a quantum (random) walk (QW). In a classical random walk on a $1 \mathrm{D}$ chain, a walker randomly decides (for instance by flipping a coin) at every step to move left or right. The QW is obtained when both the coin and the walker are quantum objects. Despite the seemingly simple setting, QW experiments have considerable potential. It has been demonstrated that the QW allows for the simulation of the topological properties of complex quantum systems ${ }^{95,96}$, for the implementation of quantum search algorithms ${ }^{97}$ and even universal quantum computation ${ }^{98}$.

In a photonic system, a quantum walk is often considered as a photon, which enters a beam splitter network with its polarization acting as the coin operation ${ }^{99,100}$. As a QW needs to be interferometrically stable (that is, the paths must be stable much below the wavelength of the photons), larger networks of interferometers are usually integrated into photonic waveguides ${ }^{101,102}$.

In 2010, Zhang et al ${ }^{103}$ showed that one could use OAM (instead of path) and polarization to perform QWs using so-called q-plates, which are devices developed by Marrucci et al ${ }^{104}$ that can couple polarization and OAM of light. Such schemes have a considerable advantage in that integrating the walker into the photonic waveguide circuits is not necessary anymore, as the phase between different OAM modes does not fluctuate during propagation (see Figure 5 (Ref. 105) for a sketch of such a setup). In 2015, Cardano et al ${ }^{106}$ have used a set of five q-plates to perform a quantum walk with 5 steps and investigated the dynamics of the propagation for both single and two-photon inputs. Other interesting applications for QW are the exploration of topological phases in $1 \mathrm{D}$ and $2 \mathrm{D}$ systems ${ }^{95}$. Those generalized geometric phases introduced by Berry ${ }^{107}$ play a key role in solid state physics,

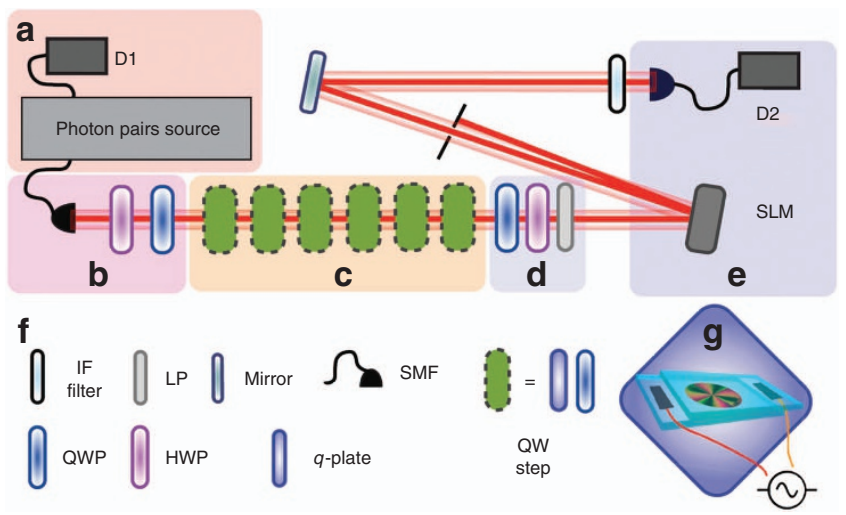

Figure 5 Sketch of a quantum walk in OAM with six steps, used in Ref. 105. (a) The source of two indistinguishable photons. (b, d) Used to control the polarization of the light before and after the QW. (c) The six steps of the quantum walk. (e) The detection system based on SLMs and single-mode fiber. (f) Legend of the elements. (g) Graphical depiction of a q-plate. Reprinted by permission from Macmillan Publishers Ltd: NATURE COMMUNICATION ${ }^{105}$, copyright 2016.

such as the description of the quantum Hall effect ${ }^{105}$ (see also Ref. 108). Cardano et al ${ }^{109}$ were also able to use their multistep q-plate system for observing a topological phase transition and topological invariants ${ }^{110}$ in a $1 \mathrm{D}$ system.

\section{Quantum teleportation of multiple-degrees of freedom of a single photon}

Quantum teleportation, proposed in 1993 by Bennet et al ${ }^{111}$, is an important quantum protocol with applications in quantum network and quantum computation. It allows Alice and Bob (if they share an entangled state) to distribute an arbitrary quantum state without sending it, just by sending classical information.

The concept is shown in Figure 6b, specifically, for a twodimensional system. There, Alice and Bob share a maximally entangled Bell state. Alice has an arbitrary quantum state $|\phi\rangle$ which she wants to transmit to Bob. She performs a Bell-state measurement 
a
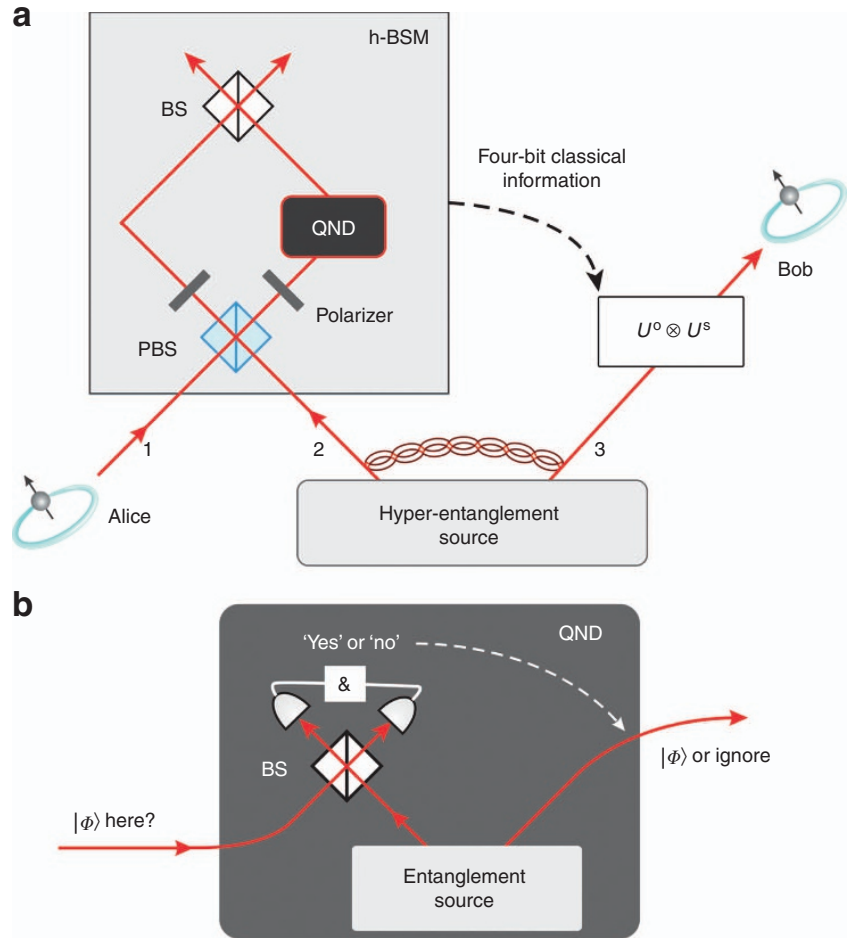

Figure 6 (a) Experimental scheme to simultaneously teleport two degrees of freedom of a single photon, as described by Ref. 112. A hyperentanglement source produces photon pairs entangled in their polarization and OAM degree-of-freedom. The polarization degree-of-freedom is teleported at the polarizing beam splitter (PBS). The two polarizers then erase the polarization information on the photons. To ensure that the Bell state measurement for the polarization teleportation succeeds, a quantum-non-demolition measurement (QND) is performed. This QND itself is again a teleportation scheme, see inset (b). Then, the OAM degree-of- freedom is teleported with a Bell-state measurement performed at the upper beam splitter. The results of both Bell-state measurements are then encoded in four classical bits and send to Bob who can perform the according unitary transformation to restore the initial quantum state from Alice. Reprinted by permission from Macmillan Publishers Ltd: NATURE 112 , copyright 2015.

between her entangled photon and $|\phi\rangle$. Then, she announces the result via a classical channel to Bob. If her result was a $\left|\phi^{+}\right\rangle$state, the initial state $|\phi\rangle$ has been transmitted to Bob successfully. If the outcome of the state measurement is different, Bob can perform a transformation to get the original $|\phi\rangle$ (or he ignores the photon which reduces the efficiency of the implementation). The first experiment implementing quantum teleportation was performed 1999 by Bouwmeester et $a l^{113}$ utilizing the polarization of photons. Since then, quantum teleportation has been performed in various different physical systems with ever improving quality—very recently even between a satellite and a ground station in China ${ }^{114}$. All of these experiments have in common that they only teleport one degree-offreedom of the quantum particle, and only in a two-dimensional system - thus only a small part of the full information of the quantum system was transferred.

A recent experiment by Wang et al ${ }^{112}$ has overcome this limitation for the first time, by showing the simultaneous teleportation of two degrees of freedom of a single photon. The basic idea behind the teleportation of multiple degrees of freedom is to successively teleport one degree after the other. In experiments so far, the incoming quantum state was destroyed during the teleportation, thus no second teleportation was possible. The clever way to get around this is to use a quantum-non-demolition measurement (QND). In this QND measurement, the quantum state is not destroyed and thus the remaining quantum information, which is stored in the other degrees of freedom, is still intact and can be teleported in the next steps. Interestingly, this QND itself is another teleportation protocol. Thus, the authors used teleportation itself as QND to enable the teleportation of a second degree-of-freedom of the particle. The measured fidelities $F=\operatorname{Tr}(\rho|\psi\rangle\langle\psi|)$ between the experimentally teleported state $\rho$ and the theoretically expected state $|\psi\rangle$ range from $0.68 \pm 0.04$ to $0.62 \pm 0.04$. The classical limit for optimal quantum state estimation of a pure state ${ }^{115}$ is given by $F_{\text {opt }}^{\mathrm{cl}}(d=4)=\frac{2}{1+d}=0.4$, where $d$ is the dimension of the system. Thus, the results show the successful implementation of a quantum teleportation scheme for a polarization-OAM composite state of a single photon.

In the future, it will be important to teleport more levels of a single high-dimensional degree-of-freedom and to use more of the available degrees-of-freedom of a single photon, in order to transmit more information stored in a quantum state.

Experimental creation of a Greenberger-Horne-Zeilinger state in three-dimensions

Three particle Greenberger-Horne-Zeilinger (GHZ) states in two dimensions were first discovered and investigated in 1989 by Greenberger-Horne-Zeilinger ${ }^{116,117}$ and were later generalized to $\mathrm{N}$ particles by Mermin ${ }^{118}$. Since their discovery, GHZ states have played an essential role in quantum information, especially in the classification of entanglement, communication complexity problems, quantum-error-correction schemes for quantum computing and also for novel experimental tests of local-realistic theories.

GHZ states have been intensively studied experimentally as well as theoretically. All experimental studies focused thereby on increasing the number of involved particles rather than increasing the dimensionality of the single particles involved. Recently, a first step towards achieving this goal was reported by entangling three particles out of which two were entangled in three dimensions, while the third particle was encoded as a qubit ${ }^{119}$. This asymmetric arrangement already shows that multipartite, high-dimensional quantum states offer novel features of entanglement ${ }^{120}$. Finally, in a very recent experiment, the first completely high-dimensional multipartite state was created. It is a generalization of GHZ state, which goes beyond qubits ${ }^{121}$.

Although, for two-dimensional GHZ states a clear recipe to increase the number of particles exists, this does not hold for increasing the dimensionality. The authors utilized the computer algorithm MEL$\mathrm{VIN}^{66}$ to find an experimental setup to create a three-dimensional GHZ state $|\psi\rangle=(|0,0,0\rangle+|1,1,1\rangle+|2,2,2\rangle) / \sqrt{ } 3$. Despite the simple correlations present in this state, the actual experimental setup, depicted in Figure 7, is neither intuitive nor simple.

The main idea is to use two sources of three-dimensional entangled photon pairs and combine them in such a way that one trigger photon heralds the 3-dimensional GHZ state in the other three photons. If the two-photon pairs are emitted at the same time, the overall four photon state shows correlations between each of the $3 \times 3=9$ terms of the quantum state. These correlations are depicted in Figure $7 \mathrm{~b}$ as red, green and blue lines. At first, there are nine connecting lines, while in the final three dimensional GHZ only three connections exist. Thus, six of the nine probability amplitudes need to be coherently suppressed in the experimental setup. This can be achieved with a three input and three output multiport. The detailed transformations of the multiport are given in Figure 7a. The basic idea behind the multiport is to utilize an even/odd OAM mode sorter ${ }^{72}$, a Hong-Ou-Mandel effect, 

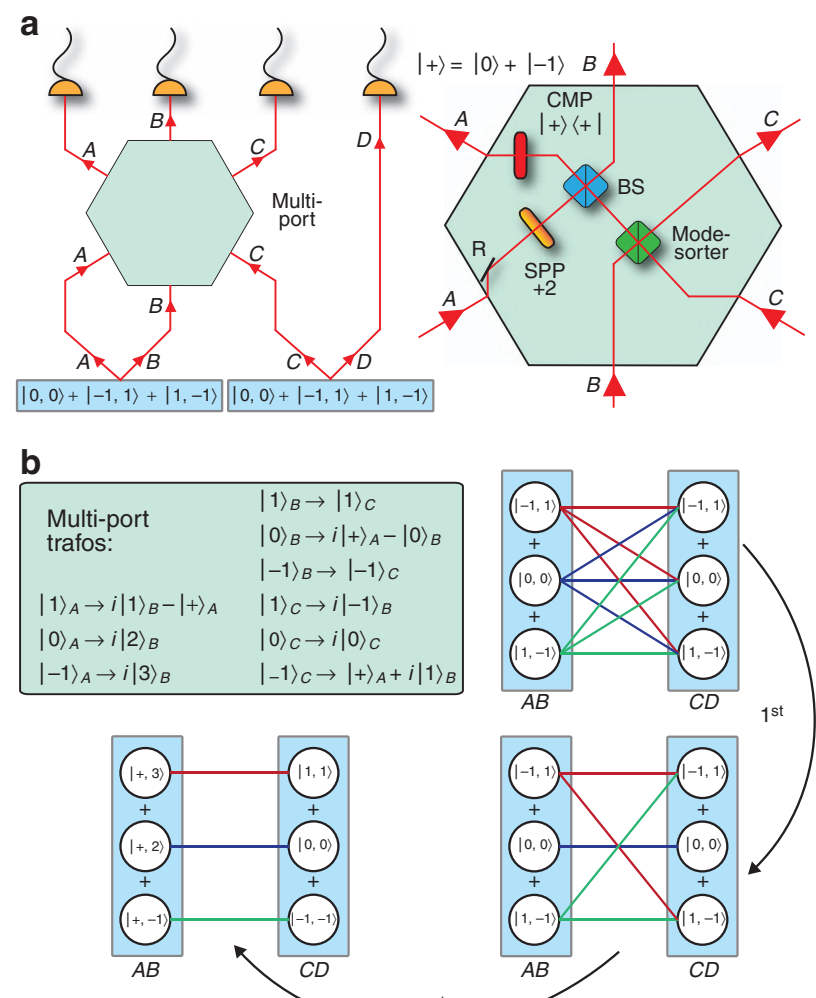

$2^{\text {nd }}$

Figure 7 (a) Experimental scheme to generate a three-photon and three dimensional GHZ state ${ }^{121}$. Two non-linear crystals each produce a pair of photons that are three dimensionally entangled. Three out of the four photons $(\mathrm{A}-\mathrm{C})$ enter a multiport which transforms each photon according to the transformations depicted in the figure. Interestingly, for each input port $(A-C)$ of the multiport a different OAM mode $|-1\rangle,|0\rangle,|1\rangle$ exits in a superposition of two different paths. The multiport consists of a reflections (R), spiral-phase-plate (SPP) that add a constant value of $\ell=2$, a mode sorter that sorts OAM modes according to their parity (even/odd), a 50/50 beam splitter (BS) and a coherent mode projection (CMP). (b) Graphical representation of the three dimensional $\mathrm{GHZ}$ state generation. If the two non-linear crystals emit a photon pair coherently at the same time, then there exist $3 \times 3=9$ probability amplitudes in total. These nine probability amplitudes are depicted by the red, blue and green connection lines. In the 1st step, the mode sorter eliminates all probability amplitudes between even and odd terms conditioned on the simultaneous detection of a photon in each detector. In the 2nd step, a Hong-Ou-Mandel two photon interference at the BS eliminates the green connection line between $|1,-1\rangle_{A B}$ and $|-1,1\rangle_{C D}$. Finally, the last remaining unwanted connection line is eliminated by rerouting the incoming photons such that no simultaneous detection event in all detectors can occur. Photon $A$ always exits the multiport in the $|D\rangle$ state and is therefore not entangled with the other three anymore. This leaves photons (B-D) in a three dimensional $\mathrm{GHZ}$ state.

a coherent mode projection and spiral phase plates (SPP) to suppress all but three possible correlations. Finally, the simultaneous detection of four photons results in a three-dimensional GHZ type entangled state for photons $\mathrm{B}, \mathrm{C}$ and $\mathrm{D}$. The experimental fidelity $F_{\exp }=$ $\operatorname{Tr}(\rho|\psi\rangle\langle\psi|)$ of $F_{\exp }=75.2 \% \pm 2.9 \%$ shows genuine three-photon and three-dimensional entanglement.

Experiments like the ones described above will allow for the investigation of the strongest form of contradictions to local realism -namely, deterministic violations of local realism as introduced by $\mathrm{GHZ}^{116}$. Generalizing GHZ violations to higher dimensions have been surprisingly difficult and has only been achieved very recently ${ }^{122-125}$. Those constructions departure significantly from their two-dimensional counterparts, and it seems as if they would help to reveal the real nature of deterministic violations of local realism.

\section{FUTURE CHALLENGES}

Finally, we will discuss a few open questions and challenges that will further advance the field of high-dimensional quantum states encoded through the OAM of photons.

\section{Long-distance distributing high-dimensional OAM states}

The undisturbed distribution of high-dimensional quantum states is one of the most important tasks for applications to extend beyond laboratory proof-of-principle demonstrations. Here, two different approaches are presented, fiber and free-space transmission, each of which face their own individual challenges.

A first step toward the fiber-based long-distance distribution of OAM states, has been achieved in developing a vortex fiber that transmits OAM states of light ${ }^{84}$. Although this fiber has been around for years, its implementation in a quantum optical setup is still yet to be demonstrated. Moreover, high-dimensional transmission is not possible, but two other approaches are conceivable: One approach would be carefully designing OAM multimode fibers, in which the modes are degenerate but do not couple to each other ${ }^{126}$. Another possibility is the fabrication of standard multimode fibers, in which intermodal coupling has to be pre or post-compensated or reduced by an appropriate choice of the modal set ${ }^{127,128}$ (recently, up to $50 \mathrm{~km}$ (Ref. 129)). As each will have its own benefits and drawbacks it will interesting to be pursuit tested in laboratories as well as real-world scenarios.

As described earlier, the second method of distributing OAM encoded qudits, namely free-space distribution, is already a step ahead. However, it is not a coincidence that the only qudit experiment was performed over a short distance (ququarts over a distance of $300 \mathrm{~m}$ (Ref. 91). While atmospheric turbulences might not completely destroy the transmitted state, even after $143 \mathrm{~km}$ (Ref. 89), it introduces considerable crosstalk between different modes, mainly due to beam wander and distortions. Therefore, adaptive optics for the correction of wave front distortions is indispensable and first steps are currently being developed ${ }^{92}$. A successful demonstration would allow for the expanding of the dimensionality of the state along with enlarging the distances over which they are distributed, maybe even between earthbased ground stations and satellites ${ }^{23,114,130}$.

Progress in these questions could lead to global quantum networks where quantum information is shared via high-dimensional quantum states encoded OAM.

\section{Quantum communication with photons in spatial modes}

So far, we have only discussed the OAM of photons. However, Laguerre-Gauss modes also have a second quantum number-the radial quantum number ${ }^{131,132}$, which can be used in quantum experiments ${ }^{133,134}$. It would be interesting to develop techniques to manipulate, detect and investigate the properties of the radial modes to enable their use in quantum communication experiments. The investigation of OAM modes in comparison with other modes would be interesting. It has been indicated that LG modes have lower crosstalk than HG modes in systems with finite apertures, thus suggesting a potential real-world advantage ${ }^{135}$. Do other mode families have different advantages? The self-healing properties of Bessel modes have been shown impressive results in the laboratory ${ }^{136}$. Can this apparent advantage also be exploited in real outdoor experiments? Do other, more complex beams have advantages in real-world quantum communication experiments-for example, Ince-Gauss modes ${ }^{137}$, which 
have only single-charged vortices in their phase profile, or Airy beams ${ }^{138}$, which tend to freely accelerate during propagation?

\section{Arbitrary transformations of OAM modes and two-qudit quantum gates}

In the Section of "Unitary transformations" three different approaches have been demonstrated that have the common goal of performing unitary transformations. While progress has been made in the last few years, it is still unclear how to perform arbitrary transformations with high fidelity and near unitary efficiency in a fast and reliable way. Such unitary transformations are required for computational tasks and their experimental demonstration might also trigger further investigations into high-dimensional computation and simulation.

Equally important for quantum computation tasks are gate operations for single and multi-particle systems. While single-qubit transformations and two-qubit gates have been studied, very little is known about high-dimensional two-qudit gates. Such gates are essential to perform the first proof-of-principle investigations of high-dimensional quantum algorithms ${ }^{54,55}$.

\section{Quantum teleportation and entanglement swapping of high- dimensional quantum states}

Another open challenge is to experimentally demonstrate highdimensional quantum teleportation and high-dimensional entanglement swapping in OAM. The experiment in the Section of "Quantum teleportation of multiple-degrees of freedom of a single photon" shows the simultaneous teleportation of two degrees of freedom of a single photon, but every degree of freedom is still two-dimensional. This problem is related to the question how to experimentally perform a higher dimensional Bell state measurement. A solution to this problem would not only allow to perform teleportation of high-dimensional quantum states encoded only in the OAM degree of freedom, but also enable other quantum protocols such as entanglement swapping, which is (together with reliable quantum memories) the key ingredient for quantum nodes in quantum networks ${ }^{139}$.

\section{Stronger violations of quantum mechanical vs. local realistic theories}

Finally, we would like to point to an open theoretical question with significant conceptual and experimental implications. Is it possible to find Bell- or Mermin-like inequalities that are more robust against noise or detection inefficiencies in higher dimensions? The known advantages for the two-photon cases have been discussed in the Section of "Larger violation of local-realistic theories and its advantages in quantum communication", but many questions remain open. Moreover, for high-dimensional multi-photon states, even less is known. The only known violations of local realism are weaker than those for two-dimensional systems ${ }^{122-125}$. In systems where both the number of photons as well as the number of dimensions is larger than two, asymmetric types of entanglement can exist ${ }^{120,140,141}$. There, the connection to violation of local realism and its applications in novel quantum protocols has not been investigated until now.

\section{CONCLUSIONS}

Within only a few years we have seen a tremendous progress in the capabilities of the generation, control and application of highdimensional quantum states with twisted photons. Among them, we have seen the first outdoor experiments distributing entanglement and high-dimensional quantum keys and we have witnessed the first generation of multi-photon entangled states and applications in quantum teleportation. These advances arose from technological progress but also from a close interaction between theory and experiment. For example, the demonstration of the novel advantages of high-dimensional systems in quantum cryptography or quantum computation are essential motivations for experiments; new theoretical classifications of multipartite high-dimensional entangled states hint to novel quantum protocols, and computer-designed experiments helping realize complex quantum states and protocols in the laboratories. We believe that the synergy between theorists and experimentalists will remain a key factor in the next few years.

To sum this up-in the spirit of Richard Feynman, What I cannot create, I do not understand-we believe it is highly desirable to continue investigating high-dimensionally encoded quantum systems -both on paper and in laboratories.

\section{CONFLICT OF INTEREST}

The authors declare no conflict of interest.

1 Schrödinger E. Die gegenwärtige situation in der quantenmechanik. Naturwissenschaften 1935; 23: 807-812.

2 Einstein A, Podolsky B, Rosen N. Can quantum-mechanical description of physical reality be considered complete? Phys Rev 1935; 47: 777-780.

3 Bell JS. On the einstein podolsky rosen paradox. Physics 1964; 1: 195-200.

4 Wootters WK, Zurek WH. A single quantum cannot be cloned. Nature 1982; 299. 802-803.

5 Ding D-S, Zhang W, Shi S, Zhou Z-Y, Li Y et al. High-dimensional entanglement between distant atomic-ensemble memories. Light Sci Appl 2016; 5: e16157, doi:10.1038/lsa.2016.157.

6 Parigi V, D'Ambrosio V, Arnold C, Marrucci L, Sciarrino F et al. Storage and retrieval of vector beams of light in a multiple-degree-of-freedom quantum memory. Nat Commun 2015; 6: 7706.

7 Kiktenko EO, Fedorov AK, Man'ko OV, Man'ko VI. Multilevel superconducting circuits as two-qubit systems: Operations, state preparation, and entropic inequalities. Phys Rev A 2015; 91: 042312.

8 Kiktenko EO, Fedorov AK, Strakhov AA, Man'ko VI. Single qudit realization of the Deutsch algorithm using superconducting many-level quantum circuits. Phys Lett $A$ 2015; 379: 1409-1413.

9 Schaeff C, Polster R, Huber M, Ramelow S, Zeilinger A. Experimental access to higher-dimensional entangled quantum systems using integrated optics. Optica 2015; 2: 523-529.

10 Martin A, Guerreiro T, Tiranov A, Designolle S, Fröwis F et al. Quantifying photonic high-dimensional entanglement. Phys Rev Lett 2017; 118: 110501.

11 Schmiegelow CT, Schulz J, Kaufmann H, Ruster T, Poschinger UG et al. Transfer of optical orbital angular momentum to a bound electron. Nat Commun 2016; 7: 12998

12 Karimi E, Schulz SA, De Leon I, Qassim H, Upham J et al. Generating optical orbital angular momentum at visible wavelengths using a plasmonic metasurface. Light Sci App/ 2014; 3: e167, doi:10.1038/Isa.2014.48.

13 Cai X, Wang J, Strain MJ, Johnson-Morris B, Zhu J et al. Integrated compact optical vortex beam emitters. Science 2012; 338: 363-366.

14 Rubinsztein-Dunlop H, Forbes A, Berry MV, Dennis MR, Andrews DL et al. Roadmap on structured light. J Opt 2016; 19: 013001.

15 Padgett MJ. Orbital angular momentum 25 years on. Opt Express 2017; 25: $11265-11274$

16 Molina-Terriza G, Torres JP, Torner L. Twisted photons. Nat Phys 2007; 3: 305-310.

17 Krenn M, Malik M, Erhard M, Zeilinger A. Orbital angular momentum of photons and the entanglement of Laguerre-Gaussian modes. Phil Trans R Soc A 2017; 375: 20150442.

18 Nielsen MA, Chuang IL. Quantum Computation and Quantum Information. Cambridge University Press, 2010.

19 Horodecki R, Horodecki P, Horodecki M, Horodecki K. Quantum entanglement. Rev Mod Phys 2009; 81: 865-942.

20 Plenio MB, Virmani S. An introduction to entanglement measures. Quantum Inf Comput 2007; 7: 1-51.

21 Bruss D. Characterizing entanglement. J Math Phys 2002; 43: 4237-4251.

22 Pan J-W, Chen Z-B, Lu C-Y, Weinfurter H, Zeilinger A et al. Multiphoton entanglement and interferometry. Rev Mod Phys 2012; 84: 777-838.

23 Yin J, Cao Y, Li Y-H, Liao S-K, Zhang L et al. Satellite-based entanglement distribution over 1200 kilometers. Science 2017; 356: 1140-1144.

24 Allen L, Beijersbergen MW, Spreeuw R, Woerdman JP. Orbital angular momentum of light and the transformation of Laguerre-Gaussian laser modes. Phys Rev A 1992; 45: 8185-8189.

25 Fickler R, Krenn M, Lapkiewicz R, Ramelow S, Zeilinger A. Real-time imaging of quantum entanglement. Sci Rep 2013; 3: 1914.

26 Bechmann-Pasquinucci H, Tittel W. Quantum cryptography using larger alphabets. Phys Rev A 2000; 61: 062308. 
27 Cerf NJ, Bourennane M, Karlsson A, Gisin N. Security of quantum key distribution using d-level systems. Phys Rev Lett 2002; 88: 127902.

28 Ekert AK. Quantum cryptography based on Bell's theorem. Phys Rev Lett 1991; 67: 661-663.

29 Bennett CH, Brassard G. Quantum Cryptography: Public Key Distribution and Coin Tossing. 1984 International Conference on Computers, Systems \& Signal Processing: Bangalore, India. 1984, 175-179.

30 Bennett $\mathrm{CH}$, Brassard G, Mermin ND. Quantum cryptography without Bell's theorem. Phys Rev Lett 1992; 68: 557-559.

31 Shor PW, Preskill J. Simple proof of security of the BB84 quantum key distribution protocol. Phys Rev Lett 2000; 85: 441-444.

32 Scarani V, Bechmann-Pasquinucci H, Cerf NJ, Dusek M, Lütkenhaus $\mathrm{N}$ et al. The security of practical quantum key distribution. Rev Mod Phys 2009; 81: 1301.

33 Bruss D. Optimal eavesdropping in quantum cryptography with six states. Phys Rev Lett 1998; 81: 3018-3021.

$34 \mathrm{Lo} \mathrm{H}$-K. Proof of unconditional security of six-state quantum key distribution scheme. Physics 2001; 1: 81-94.

35 Brádler K, Mirhosseini M, Fickler R, Broadbent A, Boyd R. Finite-key security analysis for multilevel quantum key distribution. New J Phys 2016; 18: 073030.

36 Sasaki T, Yamamoto Y, Koashi M. Practical quantum key distribution protocol without monitoring signal disturbance. Nature 2014; 509: 475-478.

37 Hensen B, Bernien H, Dréau AE, Reiserer A, Kalb $N$ et al. Loophole-free Bell inequality violation using electron spins separated by 1.3 kilometres. Nature 2015; 526: $682-686$.

38 Giustina M, Versteegh MA, Wengerowsky S, Handsteiner J, Hochrainer A et al. Significant-loophole-free test of Bell's theorem with entangled photons. Phys Rev Lett 2015; 115: 250401.

39 Shalm LK, Meyer-Scott E, Christensen BG, Bierhorst P, Wayne MA et al. Strong loophole-free test of local realism. Phys Rev Lett 2015; 115: 250402.

40 Rosenfeld W, Burchardt D, Garthoff R, Redeker K, Ortegel N et al. Event-ready bell test using entangled atoms simultaneously closing detection and locality loopholes. Phys Rev Lett 2017; 119: 010402.

41 Mermin ND. Quantum mechanics vs local realism near the classical limit: A Bell inequality for spin s. Phys Rev D 1980; 22: 356.

42 Garg A, Mermin ND. Bell inequalities with a range of violation that does not diminish as the spin becomes arbitrarily large. Phys Rev Lett 1982; 49: 901.

43 Peres A. Finite violation of a Bell inequality for arbitrarily large spin. Phys Rev A 1992; 46: 4413.

44 Kaszlikowski D, Gnaci'n ski P, Zukowski M, Miklaszewski W, Zeilinger A. Violations of local realism by two entangled $\mathrm{N}$-dimensional systems are stronger than for two qubits. Phys Rev Lett 2000; 85: 4418-4421.

45 Collins D, Gisin N, Linden N, Massar S, Popescu S. Bell inequalities for arbitrarily high-dimensional systems. Phys Rev Lett 2002; 88: 040404

46 Dada AC, Leach J, Buller GS, Padgett MJ, Andersson E. Experimental highdimensional two-photon entanglement and violations of generalized Bell inequalities. Nat Phys 2011; 7: 677-680.

47 Huber M, Pawłowski M. Weak randomness in device-independent quantum key distribution and the advantage of using high-dimensional entanglement. Phys $\operatorname{Rev} A$ 2013; 88: 032309.

48 Bouda J, Pivoluska M, Plesch M, Wilmott $C$. Weak randomness seriously limits the security of quantum key distribution. Phys Rev A 2012; 86: 062308.

49 Eberhard PH. Background level and counter efficiencies required for a loophole-free Einstein-Podolsky-Rosen experiment. Phys Rev A 1993; 47: R747-R750.

50 Giustina M, Mech A, Ramelow S, Wittmann B, Kofler J et al. Bell violation using entangled photons without the fair-sampling assumption. Nature 2013; 497: 227-230.

51 Christensen BG, McCusker KT, Altepeter JB, Calkins B, Gerrits T et al. Detectionloophole-free test of quantum nonlocality, and applications. Phys Rev Lett 2013; 111: 130406.

52 Massar S. Nonlocality, closing the detection loophole, and communication complexity. Phys Rev A 2002; 65: 032121.

53 Vértesi T, Pironio S, Brunner N. Closing the detection loophole in Bell experiments using qudits. Phys Rev Lett 2010; 104: 060401.

54 Campbell ET, Anwar H, Browne DE. Magic-state distillation in all prime dimensions using quantum reed-muller codes. Phys Rev X 2012; 2: 041021.

55 Bocharov A, Roetteler M, Svore KM. Factoring with qutrits: Shor's algorithm on ternary and metaplectic quantum architectures. Phys Rev A 2017; 96: 012306.

56 Mair A, Vaziri A, Weihs G, Zeilinger A. Entanglement of the orbital angular momentum states of photons. Nature 2001; 412: 313-316.

57 Miatto FM, Yao AM, Barnett SM. Full characterization of the quantum spiral bandwidth of entangled biphotons. Phys Rev A 2011; 83: 033816-033819.

58 Romero J, Giovannini D, Franke-Arnold S, Barnett SM, Padgett MJ. Increasing the dimension in high-dimensional two-photon orbital angular momentum entanglement. Phys Rev A 2012; 86: 012334.

59 Chen L, Romero J. Hardy's nonlocality proof using twisted photons. Opt Express 2012; 20: 21687-21692.

60 Chen L, Zhang W, Wu Z, Wang J, Fickler R et al. Experimental ladder proof of Hardy's nonlocality for high-dimensional quantum systems. Phys Rev A 2017; 96: 022115.

61 Zhang Y, Roux FS, Konrad T, Agnew M, Leach J et al. Engineering two-photon highdimensional states through quantum interference. Sci Adv 2016; 2: e1501165.

62 Hong CK, Ou Z-Y, Mandel L. Measurement of subpicosecond time intervals between two photons by interference. Phys Rev Lett 1987; 59: 2044-2046.
63 Fickler R, Lapkiewicz R, Huber M, Lavery MP, Padgett MJ et al. Interface between path and OAM entanglement for high-dimensional photonic quantum information. Nat Commun 2014; 26: 060305.

64 Krenn M, Hochrainer A, Lahiri M, Zeilinger A. Entanglement by Path Identity. Phys Rev Lett 2017; 118: 080401

65 Krenn M, Gu X-M, Zeilinger A. Quantum experiments and graphs: multiparty states as coherent superpositions of perfect matchings. Phys Rev Lett 2017; 119: 240403.

66 Krenn M, Malik M, Fickler R, Lapkiewicz R, Zeilinger A. Automated search for new quantum experiments. Phys Rev Lett 2016; 116: 090405.

67 Carolan J, Harrold C, Sparrow C, Martín-López E, Russell NJ et al. Universal linear optics. Science 2015; 349: 711-716.

68 Morizur J-F, Nicholls L, Jian P, Armstrong S, Treps N et al. Programmable unitary spatial mode manipulation. J Opt Soc Am A 2010; 27: 2524-2531.

69 Fickler R, Ginoya M, Boyd RW. Custom-tailored spatial mode sorting by controlled random scattering. Phys Rev B 2017; 95: 161108.

70 Asadian A, Erker P, Huber M, Klöckl C. Heisenberg-Weyl observables: Bloch vectors in phase space. Phys Rev A 2016; 94: 010301.

71 Babazadeh A, Erhard M, Wang F, Malik M, Nouroozi R et al. High-dimensional singlephoton quantum gates: concepts and experiments. Phys Rev Lett 2017; 119: 180510.

72 Leach J, Padgett MJ, Barnett SM, Franke-Arnold S, Courtial J. Measuring the orbital angular momentum of a single photon. Phys Rev Lett 2002; 88: 257901.

73 de Oliveira AN, Walborn SP, Monken $\mathrm{CH}$. Implementing the Deutsch algorithm with polarization and transverse spatial modes. J Opt B Quantum Semiclass Opt 2005; 7: 288.

74 Chen D-X, Liu R-F, Zhang P, Wang Y-L. Realization of quantum permutation algorithm in high dimensional Hilbert space. Chin Phys B 2017; 26: 060305.

75 Schlederer F, Krenn M, Fickler R, Malik M, Zeilinger A. Cyclic transformation of orbital angular momentum modes. New J Phys 2016; 18: 043019.

76 Scarani V, Iblisdir S, Gisin N, Acin A. Quantum cloning. Rev Mod Phys 2005; 77: 1225-1256.

77 Nagali E, Giovannini D, Marrucci L, Slussarenko S, Santamato E et al. Experimental optimal cloning of four-dimensional quantum states of photons. Phys Rev Lett 2010; 105: 073602

78 Bouchard F, Fickler R, Boyd RW, Karimi E. High-dimensional quantum cloning and applications to quantum hacking. Sci Adv 2017; 3: e1601915.

79 Nagali E, Sansoni L, Sciarrino F, De Martini F, Marrucci L et al. Optimal quantum cloning of orbital angular momentum photon qubits through Hong-Ou-Mandel coalescence. Nat Photonics 2009; 3: 720-723.

80 Gröblacher S, Jennewein T, Vaziri A, Weihs G, Zeilinger A. Experimental quantum cryptography with qutrits. New J Phys 2006; 8: 75.

81 Mafu M, Dudley A, Goyal S, Giovannini D, McLaren M, Padgett MJ et al. Higherdimensional orbital-angular-momentum-based quantum key distribution with mutually unbiased bases. Phys Rev A 2013; 88: 032305.

82 Mirhosseini M, Malik M, Shi Z, Boyd RW. Efficient separation of the orbital angular momentum eigenstates of light. Nat Commun 2013; 4: 2781.

83 Vallone G, D'Ambrosio V, Sponselli A, Slussarenko S, Marrucci L et al. Free-space quantum key distribution by rotation-invariant twisted photons. Phys Rev Lett 2014; 113: 060503

84 Bozinovic N, Yue Y, Ren Y, Tur M, Kristensen P et al. Terabit-scale orbital angular momentum mode division multiplexing in fibers. Science 2013; 340: 1545-1548.

85 Gibson G, Courtial J, Padgett MJ, Vasnetsov M, Pas'ko V et al. Free-space information transfer using light beams carrying orbital angular momentum. Opt Express 2004; 12: 5448-5456.

86 Wang J, Yang J-Y, Fazal IM, Ahmed N, Yan Y et al. Terabit free-space data transmission employing orbital angular momentum multiplexing. Nat Photonics 2012; 6: 488-496.

87 Krenn M, Fickler R, Fink M, Handsteiner J, Malik M et al. Communication with spatially modulated light through turbulent air across Vienna. New J Phys 2014; 16: 113028.

88 Ren Y, Wang Z, Liao P, Li L, Xie G et al. Experimental characterization of a 400 Gbit/s orbital angular momentum multiplexed free-space optical link over $120 \mathrm{~m}$. Opt Lett 2016; 41: 622-625.

89 Krenn M, Handsteiner J, Fink M, Fickler R, Ursin R et al. Twisted light transmission over 143 km. Proc Natl Acad Sci USA 2016; 113: 13648-13653.

90 Krenn M, Handsteiner J, Fink M, Fickler R, Zeilinger A. Twisted photon entanglement through turbulent air across Vienna. Proc Natl Acad Sci USA 2015; 112: 14197-14201.

91 Sit A, Bouchard F, Fickler R, Gagnon-Bischoff J, Larocque H et al. High-dimensional intracity quantum cryptography with structured photons. Optica 2017; 9: 1006-1010

92 Ren Y, Xie G, Huang H, Ahmed N, Yan Y et al. Adaptive-optics-based simultaneous pre-and post-turbulence compensation of multiple orbital-angular-momentum beams in a bidirectional free-space optical link. Optica 2014; 1: 376-382.

93 Hughes RJ, Nordholt JE, Derkacs D, Peterson CG. Practical free-space quantum key distribution over $10 \mathrm{~km}$ in daylight and at night. New J Phys 2002; 4: 43.

94 Liao S-K, Yong H-L, Liu C, Shentu G-L, Li D-D et al. Long-distance free-space quantum key distribution in daylight towards inter-satellite communication. Nat Photonics 2017; 11: nphoton-2017.

95 Kitagawa T, Rudner MS, Berg E, Demler E. Exploring topological phases with quantum walks. Phys Rev A 2010; 82: 033429. 
96 Kitagawa T, Broome MA, Fedrizzi A, Rudner MS, Berg E et al. Observation of topologically protected bound states in photonic quantum walks. Nat Commun 2016; 3: 882

97 Shenvi N, Kempe J, Whaley KB. Quantum random-walk search algorithm. Phys Rev $A$ 2003; 67: 052307.

98 Childs AM. Universal computation by quantum walk. Phys Rev Lett 2009; 102 : 180501.

99 Broome MA, Fedrizzi A, Lanyon BP, Kassal I, Aspuru-Guzik A et al. Discrete singlephoton quantum walks with tunable decoherence. Phys Rev Lett 2010; 104: 153602.

100 Schreiber A, Cassemiro KN, Potoček V, Gábris A, Mosley PJ et al. Photons walking the line: a quantum walk with adjustable coin operations. Phys Rev Lett 2010; 104: 050502.

101 Peruzzo A, Lobino M, Matthews JC, Matsuda N, Politi A et al. Quantum walks of correlated photons. Science 2010; 329: 1500-1503.

102 Sansoni L, Sciarrino F, Vallone G, Mataloni P, Crespi A et al. Two-particle bosonicfermionic quantum walk via integrated photonics. Phys Rev Lett 2012; 108: 010502.

103 Zhang P, Liu B-H, Liu R-F, Li H-R, Li F-L et al. Implementation of one-dimensional quantum walks on spin-orbital angular momentum space of photons. Phys $\operatorname{Rev} A$ 2010; 81: 052322

104 Marrucci L, Manzo C, Paparo D. Optical spin-to-orbital angular momentum conversion in inhomogeneous anisotropic media. Phys Rev Lett 2006; 96: 163905.

105 Thouless DJ, Kohmoto M, Nightingale MP, Nijs Den M. Quantized Hall conductance in a two-dimensional periodic potential. Phys Rev Lett 1982; 49: 405-408.

106 Cardano F, Massa F, Qassim H, Karimi E, Slussarenko S et al. Quantum walks and wavepacket dynamics on a lattice with twisted photons. Sci Adv 2015; 1: e1500087.

107 Berry MV. Quantal phase factors accompanying adiabatic changes. Proc R Soc Lond A Math Phys Sci 1984; 392: 45-57.

108 Lu L, Joannopoulos JD, Soljačić M. Topological states in photonic systems. Nat Phys 2016; 12: 626-629.

109 Cardano F, Maffei M, Massa F, Piccirillo B, De Lisio C et al. Statistical moments of quantum-walk dynamics reveal topological quantum transitions. Nat Commun 2016; 7: 11439

110 Cardano F, D'Errico A, Dauphin A, Maffei M, Piccirillo B et al. Detection of Zak phases and topological invariants in a chiral quantum walk of twisted photons. Nat Commun 2017; 8: 15516.

111 Bennett CH, Brassard G, Crépeau C, Jozsa R, Peres A et al. Teleporting an unknown quantum state via dual classical and Einstein-Podolsky-Rosen channels. Phys Rev Lett 1993; 70: 1895-1899.

112 Wang X-L, Cai X-D, Su Z-E, Chen M-C, Wu D et al. Quantum teleportation of multiple degrees of freedom of a single photon. Nature 2015; 518: 516-519.

113 Bouwmeester D, Pan JW, Mattle K, Eibl M, Weinfurter H. Experimental quantum teleportation. Nature 1997; 390: 575-579.

114 Ren JG, Xu P, Yong HL, Zhang L, Liao SK et al. Ground-to-satellite quantum teleportation. Nature advance online publication 2017; doi:(101038/nature23675). 2017.

115 Hayashi A, Hashimoto T, Horibe M. Reexamination of optimal quantum state estimation of pure states. Phys Rev A 2005; 72: 032325.

116 Greenberger DM, Horne MA, Zeilinger A. Going beyond Bell's theorem. In: Kafatos M, editor. Bell's Theorem, Quantum Theory and Conceptions of the Universe. New York: Springer; 1989. p 69-72. arXiv:0712.0921.

117 Greenberger DM, Horne MA, Shimony A, Zeilinger A. Bell's theorem without inequalities. Am J Phys 1990; 58: 1131-1143.

118 Mermin ND. Extreme quantum entanglement in a superposition of macroscopically distinct states. Phys Rev Lett 1990; 65: 1838-1840.

119 Malik M, Erhard M, Huber M, Krenn M, Fickler R et al. Multi-photon entanglement in high dimensions. Nat Photonics 2017; 10: 248-252.

120 Huber M, de Vicente Jl. Structure of multidimensional entanglement in multipartite systems. Phys Rev Lett. 2013; 110: 030501-030505.
121 Erhard M, Malik M, Krenn M, Zeilinger A. Experimental GHZ Entanglement beyond Qubits. arXiv preprint arXiv:170803881. 2017.

122 Ryu J, Lee C, Z ukowski M, Lee J. Greenberger-Horne-Zeilinger theorem for N qudits. Phys Rev A 2013; 88: 042101.

123 Ryu J, Lee C, Yin Z, Rahaman R, Angelakis DG et al. Multisetting Greenberger-HorneZeilinger theorem. Phys Rev A 2014; 89: 024103.

124 Lawrence J. Rotational covariance and Greenberger-Horne-Zeilinger theorems for three or more particles of any dimension. Phys Rev A 2014; 89: 012105.

125 Lawrence J. Mermin inequalities for perfect correlations in many-qutrit systems. Phys Rev A 2017; 95: 042123

126 Gregg $\mathrm{P}$, Kristensen $\mathrm{P}$, Ramachandran S. Conservation of orbital angular momentum in air-core optical fibers. Optica 2015; 2: 267-270.

127 Huang H, Milione G, Lavery MPJ, Xie G, Ren Y et al. Mode division multiplexing using an orbital angular momentum mode sorter and MIMO-DSP over a graded-index fewmode optical fibre. Sci Rep 2015; 5: 14931.

128 Carpenter J, Eggleton BJ, Schröder J. Observation of Eisenbud-Wigner-Smith states as principal modes in multimode fibre. Nat Photonics 2015; 9: 751-757.

129 Wang A, Zhu L, Chen S, Du C, Mo Q et al. Characterization of LDPC-coded orbital angular momentum modes transmission and multiplexing over a 50-km fiber. Opt Express 2016; 24: 11716-11726.

130 Liao S-K, Cai W-Q, Liu W-Y, Zhang L, Li Y et al. Satellite-to-ground quantum key distribution. Nature 2017; 549: 43-47.

131 Karimi E, Boyd RW, la Hoz de P, de Guise H, Řeháček J et al. Radial quantum number of Laguerre-Gauss modes. Phys Rev A 2014; 89: 063813.

132 Plick WN, Krenn M. Physical meaning of the radial index of Laguerre-Gauss beams. Phys Rev A 2015; 92: 063841.

133 Karimi E, Giovannini D, Bolduc E, Bent N, Miatto FM et al. Exploring the quantum nature of the radial degree of freedom of a photon via Hong-Ou-Mandel interference. Phys Rev A 2014; 89: 013829-0138355.

134 Krenn M, Huber M, Fickler R, Lapkiewicz R, Ramelow S et al. Generation and confirmation of a $(100 \times 100)$-dimensional entangled quantum system. Proc Natl Acad Sci USA 2014; 111: 6243-6247.

135 Restuccia S, Giovannini D, Gibson G, Padgett M. Comparing the information capacity of Laguerre-Gaussian and Hermite-Gaussian modal sets in a finite-aperture system. Opt Express 2016; 24: 27127-27136.

136 McLaren M, Mhlanga T, Padgett MJ, Roux FS, Forbes A. Self-healing of quantum entanglement after an obstruction. Nat Commun 2014; 5: 3248.

137 Krenn M, Fickler R, Huber M, Lapkiewicz R, Plick W et al. Entangled singularity patterns of photons in Ince-Gauss modes. Phys Rev A 2013; 87: 012326.

138 Siviloglou GA, Broky J, Dogariu A, Christodoulides DN. Observation of accelerating Airy beams. Phys Rev Lett 2007; 99: 213901.

139 Duan L-M, Lukin M, Cirac I, Zoller P. Long-distance quantum communication with atomic ensembles and linear optics. Nature 2001; 414: 413-418.

140 Huber M, Perarnau-Llobet M, de Vicente Jl. Entropy vector formalism and the structure of multidimensional entanglement in multipartite systems. Phys Rev $A$ 2013; 88: 042328-042337.

141 Goyeneche D, Bielawski J, Z yczkowski K. Multipartite entanglement in heterogeneous systems. Phys Rev A 2016; 94: 012346.

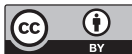

This work is licensed under a Creative Commons Attribution 4.0 International License. The images or other third party material in this article are included in the article's Creative Commons license, unless indicated otherwise in the credit line; if the material is not included under the Creative Commons license, users will need to obtain permission from the license holder to reproduce the material. To view a copy of this license, visit http://creativecommons.org/licenses/by/4.0/

(C) The Author(s) 2018 